\title{
EXISTENCE OF A SOLUTION FOR THE FRACTIONAL FORCED PENDULUM
}

\author{
César Torres \\ Departamento de Ingeniería Matemática, Centro de Modelamiento Matemático \\ Universidad de Chile, Santiago, Chile \\ ctorres@dim.uchile.cl
}

Abstract. In this work we study the fractional forced pendulum equation with combined fractional derivatives

$$
\begin{gathered}
-{ }_{t} D_{T 0}^{\alpha} D_{t}^{\alpha} u(t)+g(u(t))=f(t), \quad t \in(0, T) \\
u(0)=u(T)=0
\end{gathered}
$$

where $\frac{1}{2}<\alpha<1, g \in C(\mathbb{R}, \mathbb{R})$, bounded and $f \in C[0, T]$. Using minimization techniques form variational calculus we show that $(0.1)$ has a nontrivial solution.

Keywords: fractional calculus, fractional boundary value problem, mountain pass theorem, fractional space

\section{Introduction}

Fractional order models can be found to be more adequate than integer order models in some real world problems as fractional derivatives provide an excellent tool for the description of memory and hereditary properties of various materials and processes. The mathematical modeling of systems and processes in the fields of physics, chemistry, aerodynamics, electrodynamics of complex medium, polymer rheology, etc. involves derivatives of fractional order. As a consequence, the subject of fractional differential equations is gaining more importance and attention. There has been significant development in ordinary and partial differential equations involving both Riemann-Liouville and Caputo fractional derivatives. For details and examples, one can see the monographs [1-3] and the papers [4-13].

Recently, also equations including both - left and right fractional derivatives, are discussed. Equations of this type are known in literature as the fractional Euler-Lagrange equation and are obtained by modifying the principle of least action and applying the rule of fractional integration by parts. The first results were derived by Riewe $[14,15]$. Then the Lagrangian and the Hamiltonian formulation of fractional mechanics were developed for models with a symmetric and an antisymmetric fractional derivative [16], subsequently for models with sequential fractional derivatives [17] and for models with constraints [18]. 
The characteristic feature of these equations of motion is the mixing of left- and right-sided Riemann-Liouville fractional derivatives. Therefore, these new classes of fractional differential equations become an interesting area of investigation. Certain fractional equations of this type were studied in papers [19-22]. In the solution of fractional equations of variational type the composition rules of fractional calculus together with fixed point theorems were applied. Unfortunately, this solution is represented by series of alternately left and right fractional integrals and therefore is difficult in any practical calculations. Then the Mellin transform was proposed as a method of solving some equations including the composition of left- and right-sided derivatives [23], but this solution is represented by complicated series of special functions. This greatly limits practical implementations, for example sometimes it is very difficult to illustrate the solution in a graphical form.

We note, in particular, that the fractional oscillator equation with mixed derivatives

$$
{ }_{t} D_{b}^{\alpha}{ }_{a} D_{t}^{\alpha} u(t)=\lambda u(t), \quad t \in[a, b]
$$

and the nonlinear version

$$
\left({ }_{t} D_{T 0}^{\alpha} D_{t}^{\alpha}-\lambda\right) u(t)+V^{\prime}(u(t))=0, \quad t \in[a, b]
$$

were studied in the works mentioned above. This type of equations has been found very useful tools for modelling many phenomena in the construction industry $[24,25]$.

Motivated by these previous works, in this paper we deal with the fractional forced pendulum equation

$$
\begin{gathered}
-{ }_{t} D_{T \quad 0}^{\alpha} D_{t}^{\alpha} u(t)+g(u(t))=f(t), \quad t \in(0, T) \\
u(0)=u(T)=0
\end{gathered}
$$

where $\frac{1}{2}<\alpha<1, g \in C(\mathbb{R}, \mathbb{R})$, bounded and $f \in C[0, T]$.

In particular, if $\alpha=1$, boundary value problem (1.1) reduces to the standard second order forced pendulum equation

$$
u^{\prime \prime}(t)+g(u(t))=f(t)
$$

Hamel was the first researcher who considered this problem in a particular case: $g(u(t))=a \sin (u(t))$ and $f(t)=b \sin (t)$. Hamel's paper starts by an existence result for a $2 \pi$-periodic solution of equation (1.2) by using the direct method of the calculus of variations were made rigorously by Hilbert at the beginning of the century. After Hamel's work had been a great interest in study existence of periodic solution to (1.2) and its generalization see [26] and [27]. 
Before stating our main results, let us introduce the main ingredients involved in our approach. We define the fractional space $E^{\alpha}=\left\{u \in L^{2}[0, T]:{ }_{0} D_{t}^{\alpha} u \in L^{2}[0, T]\right.$ and $u(0)=u(T)=0\}$ and we say that $u$ is a weak solution of (1.1) if

$$
\int_{0}^{T}\left[-{ }_{0} D_{t}^{\alpha} u(t){ }_{0} D_{t}^{\alpha} v(t)+g(u(t)) v(t)\right] d t=\int_{0}^{T} f(t) v(t) d t, \quad \forall v \in E^{\alpha}
$$

Moreover, we note that $u$ is a weak solution of (1.3) if and only if $u$ is a critical point of the functional

$$
I(u)=\int_{0}^{T}\left[\frac{\left|{ }_{0} D_{t}^{\alpha} u(t)\right|^{2}}{2}-G(u(t))+f(t) u(t)\right] d t
$$

which is $C^{1}$, weakly-lower semicontinuous and satisfies the $(P S)_{C}$ condition. Now we are in a position to state our main existence theorem.

Theorem 1.1. Let $\frac{1}{2}<\alpha<1, g \in C(\mathbb{R}, \mathbb{R})$, bounded and $f \in C[0, T]$. The problem (1.3) has at least one solution.

Our approach to prove Theorem 1.1 is variational. We used to classical result from the calculus of variation. We recall this theorem for the reader's convenience.

Theorem 1.2. [28] Let $\varphi$ be a weakly lower semi-continuous functional bounded from below on the reflexive Banach space $X$. If $\varphi$ is coercive, then $c=i n f_{X} \varphi$ is attained at a point $x_{0} \in X$.

Theorem 1.3. [28] Let $\varphi \in C^{1}(X, \mathbb{R})$ be a bounded below and $c=i n f_{X} \varphi$. Assume that $\varphi$ satisfies $(P S)_{C}$ condition. Then $\mathbf{c}$ is achieved at a point $x_{0} \in X$ and

$$
\varphi^{\prime}\left(x_{0}\right)=0 .
$$

Where a functional $\varphi \in C^{1}(X, \mathbb{R})$ satisfies the Palais-Smale (PS) condition if every sequence $x_{n} \in X$ such that $\varphi\left(x_{n}\right)$ is bounded and $\lim _{n \rightarrow \infty} \varphi^{\prime}\left(x_{n}\right)=0$ in $X^{*}$ has a convergent subsequence. A variant of (PS) condition, noted as $(P S)_{C}$ was introduced by Brézis, Coron and Niremberg [28]: Let $c \in \mathbb{R}, \varphi \in C^{1}(X, \mathbb{R})$ satisfies the $(P S)_{C}$ condition if every sequence $x_{n} \in X$ such that

$$
\lim _{n \rightarrow \infty} \varphi\left(x_{n}\right)=c \text { and } \lim _{n \rightarrow \infty} \varphi^{\prime}\left(x_{n}\right)=0 \text { in } X^{*},
$$

has a convergent subsequence. It is clear that (PS) condition implies the $(P S)_{C}$ condition for every $c \in \mathbb{R}$.

This article is organized as follows. In $\S 2$ we present preliminaries on fractional calculus and we introduce the functional setting of the problem (1.3). In $\S 3$ we prove the Theorem 1.1. 


\section{Reminder about fractional calculus}

\subsection{Some spaces of functions}

For any $p \geq 1, L_{p}:=L_{p}(a, b)$ denotes the classical Lebesgue space of $p$-integrable functions endowed with its usual norm $\|\cdot\|_{p}$. Let us give some usual notations of spaces of continuous functions defined on $[a, b]$ with values in $R$ :

- $A C:=A C[a, b]$ the space of absolutely continuous functions;

- $C^{\infty}:=C^{\infty}[a, b]$ the space of infinitely differentiable functions;

- $C_{0}^{\infty}:=C_{0}^{\infty}[a, b]$ the space of infinitely differentiable functions and compactly supported in $(a, b)$.

We remind that a function $f \in A C$ if and only if $f^{\prime} \in L_{1}$ and the following equality holds:

$$
\forall t \in[a, b], \quad f(t)=f(a)+\int_{a}^{t} f^{\prime}(s) d s
$$

where $f^{\prime}$ denotes the derivative of $f$. Finally, we denote by $C_{a}$ (resp. $A C_{a}$ or $C_{a}^{\infty}$ ) the space of functions $f \in C$ (resp. $A C$ or $C^{\infty}$ ) such that $f(a)=0$. In particular,

$$
C_{0}^{\infty} \subset C_{a}^{\infty} \subset A C_{a}
$$

\subsection{Fractional calculus operators}

Let $\alpha>0$ and $u$ be a function defined a.e. on $(a, b)$ with values in $R$. The left (resp. right) fractional integral in the sense of Riemann-Liouville with inferior limit $a$ (resp. superior limit $b$ ) of order $\alpha$ of $u$ is given by:

$$
{ }_{a} I_{t}^{\alpha} u(t)=\frac{1}{\Gamma(\alpha)} \int_{a}^{t}(t-s)^{\alpha-1} u(s) d s, \forall t \in(a, b]
$$

respectively:

$$
{ }_{t} I_{b}^{\alpha} u(t)=\frac{1}{\Gamma(\alpha)} \int_{t}^{b}(s-t)^{\alpha-1} u(s) d s, \forall t \in[a, b)
$$

where $\Gamma$ denotes Euler's Gamma function. If $u \in L_{1}$, then ${ }_{a} I_{t}^{\alpha} u$ and ${ }_{t} I_{b}^{\alpha} u$ are defined a.e. on $(a, b)$.

Now, let us consider $0<\alpha<1$. The left (resp. right) fractional derivative in the sense of Riemann-Liouville with inferior limit $a$ (resp. superior limit $b$ ) of order $\alpha$ of $u$ is given by:

$$
{ }_{a} D_{t}^{\alpha} u(t)=\frac{d}{d t}\left({ }_{a} I_{t}^{1-\alpha} u\right)(t), \quad \forall t \in(a, b]
$$


respectively:

$$
{ }_{t} D_{b}^{\alpha} u(t)=-\frac{d}{d t}\left({ }_{t} I_{b}^{1-\alpha} u\right)(t), \quad \forall t \in[a, b)
$$

From [1], if $u \in A C$, then ${ }_{a} D_{t}^{\alpha} u$ and ${ }_{t} D_{b}^{\alpha} u$ are defined $a . e$. on $(a, b)$ and satisfy:

$$
{ }_{a} D_{t}^{\alpha} u(t)={ }_{a} I_{t}^{1-\alpha} u^{\prime}(t)+\frac{u(a)}{(t-a)^{\alpha} \Gamma(1-\alpha)}
$$

and

$$
{ }_{t} D_{b}^{\alpha} u(t)=-{ }_{t} I_{b}^{1-\alpha} u^{\prime}(t)+\frac{u(b)}{(b-t)^{\alpha} \Gamma(1-\alpha)}
$$

In particular, if $u \in A C_{a}$, then ${ }_{a} D_{t}^{\alpha} u(t)={ }_{a} I_{t}^{1-\alpha} u^{\prime}(t)$. So in this case we have the equality of Riemann-Liouville fractional derivative and Caputo derivative defined by

$$
{ }_{a}^{c} D_{t}^{\alpha} u(t)={ }_{a} I_{t}^{1-\alpha} u^{\prime}(t)
$$

and

$$
{ }_{t}^{c} D_{b}^{\alpha} u(t)=-{ }_{t} I_{b}^{1-\alpha} u^{\prime}(t)
$$

So with this definition (2.6) and (2.7) can be rewritten

$$
{ }_{a} D_{t}^{\alpha} u(t)={ }_{a}^{c} D_{t}^{\alpha} u(t)+\frac{u(a)}{(t-a)^{\alpha} \Gamma(1-\alpha)}
$$

and

$$
{ }_{t} D_{b}^{\alpha} u(t)={ }_{t}^{c} D_{b}^{\alpha} u(t)+\frac{u(b)}{(b-t)^{\alpha} \Gamma(1-\alpha)}
$$

\subsection{Some properties of fractional calculus operators}

In this section we provide some properties concerning the left fractional operators of Riemann-Liouville. One can easily derive the analogous version for the right ones. The first result yields the semi-group property of the left Riemann-Liouville fractional integral:

Property 2.1. For any $\alpha, \beta>0$ and any function $u \in L_{1}$, the following equality holds:

$$
{ }_{a} I_{t}^{\alpha} \circ{ }_{a} I_{t}^{\beta} u={ }_{a} I_{t}^{\alpha+\beta} u
$$


From Property 2.1 and the equations (2.6) and (2.7), one can easily deduce the following results concerning the composition between fractional integral and fractional derivative. For any $0<\alpha<1$, the following equalities hold:

$$
\forall u \in L^{1}, \quad{ }_{a} D_{t}^{\alpha} \circ{ }_{a} I_{t}^{\alpha} u=u
$$

and

$$
\forall u \in A C_{a}, \quad{ }_{a} I_{t}^{\alpha} \circ{ }_{a} D_{t}^{\alpha} u=u
$$

Another classical result is the boundedness of the left fractional integral from $L_{p}$ to $L_{p}$ :

Property 2.2. For any $\alpha>0$ and any $p \geq 1,{ }_{a} I_{t}^{\alpha} u$ is linear and continuous from $L_{p}$ to $L_{p}$. Precisely, the following inequality holds:

$$
\forall u \in L^{p},\left\|{ }_{a} I_{t}^{\alpha} u\right\|_{L^{p}} \leq \frac{(b-a)^{\alpha}}{\Gamma(1+\alpha)}\|u\|_{L^{p}}
$$

Property 2.3. (Integration by parts) Let $0<\alpha<1$. Let $u \in L_{p}, v \in L_{q}$, where

$$
p \geq 1, q \geq 1 \text { and } \frac{1}{p}+\frac{1}{q}<1+\alpha
$$

or

$$
p \neq 1, q \neq 1 \text { and } \frac{1}{p}+\frac{1}{q}=1+\alpha
$$

Then, the following equality holds:

$$
\int_{a}^{b}\left[{ }_{a} I_{t}^{\alpha} u(t)\right] v(t) d t=\int_{a}^{b} u(t)_{t} I_{b}^{\alpha} v(t) d t
$$

In the discussion to follow, we will also need the following formulae for fractional integration by parts

Property 2.4. Let $0<\alpha<1$, then

$$
\int_{a}^{b} u(t){ }_{a}^{c} D_{t}^{\alpha} v(t) d t=\left.v(t){ }_{t} I_{b}^{1-\alpha} u(t)\right|_{t=a} ^{t=b}+\int_{a}^{b} v(t){ }_{t} D_{b}^{\alpha} u(t) d t
$$

Moreover, if $v$ is a function such that $v(a)=v(b)=0$, we have simpler formulae:

$$
\int_{a}^{b} u(t){ }_{a}^{c} D_{t}^{\alpha} v(t) d t=\int_{a}^{b} v(t){ }_{t} D_{b}^{\alpha} u(t) d t
$$


The following property completes Property 2.2 in the case $0<\frac{1}{p}<\alpha<1$ : indeed, in this case, ${ }_{a} I_{t}^{\alpha} u$ is additionally bounded from $L_{p}$ to $C_{a}$ :

Property 2.5. [29] Let $0<\frac{1}{p}<\alpha<1$ and $q=\frac{p}{p-1}$. Then, for any $u \in L_{p}$, we have:

- ${ }_{a} I_{t}^{\alpha} u$ is Hölder continuous on $(a, b]$ with exponent $\alpha-\frac{1}{p}>0$;

- $\lim _{t \rightarrow a} I_{t}^{\alpha} u(t)=0$.

Consequently, ${ }_{a} I_{t}^{\alpha} u$ can be continuously extended by 0 in $t=a$. Finally, for any $u \in L^{p}$, we have ${ }_{a} I_{t}^{\alpha} u \in C_{a}$. Moreover, the following inequality holds:

$$
\forall u \in L^{p}, \quad\left\|I_{t}^{\alpha} u\right\|_{\infty} \leq \frac{(b-a)^{\alpha-1 / p}}{\Gamma(\alpha)((a-1) q+1)^{1 / q}}\|u\|_{L^{p}}
$$

\subsection{Fractional derivative space}

In order to prove the existence of a weak solution of (1.3) using a variational method, we need the introduction of an appropriate space of functions. This space has to present some properties like reflexivity, see [30].

Throughout this paper, we denote by the norm of the space $L^{p}[0, T]$ for $1 \leq p \leq+\infty$ as $\|u\|_{L^{p}}=\left(\int_{0}^{T}|u(t)|^{p} d t\right)^{1 / p}$ and $\|u\|_{\infty}=\max _{t \in[0, T]}|u(t)|$.

Definition 2.1. Let $0<\alpha<1$ and $1<p<\infty$. The fractional derivative space $E^{\alpha, p}$ is defined by

$$
E^{\alpha, p}=\left\{u:[0, T] \rightarrow \mathbb{R}: \text { uis absolutely continuous and }{ }_{0}^{c} D_{t}^{\alpha} u \in L^{p}[0, T]\right\}
$$

For every $u \in E^{\alpha, p}$, we define

$$
\|u\|_{\alpha, p}=\left(\int_{0}^{T}|u(t)|^{p} d t+\int_{0}^{T}\left|{ }_{0}^{c} D_{t}^{\alpha} u(t)\right|^{p} d t\right)^{1 / p}
$$

Definition 2.2. Let $0<\alpha \leq 1$ and $1<p<\infty$. The fractional derivative space $E^{\alpha, p}$ is defined by the closure of $C^{\infty}[0, T]$ with respect to the norm (2.18), that is

$$
E_{0}^{\alpha, p}=\overline{C_{0}^{\infty}[0, T]^{\|,\|}}
$$




\section{Remark 2.1.}

i. It is obvious that this fractional derivative space $E_{0}^{\alpha, p}$ is equal to

$$
E_{0}^{\alpha, p}=\left\{u \in L^{p}[0, T]:{ }_{0}^{c} D_{t}^{\alpha} u \in L^{p}[0, T] \text { and } u(0)=u(T)=0\right\}
$$

ii. It follows from the boundary condition $u(0)=u(T)=0$ that we see the fact that ${ }_{0}^{c} D_{t}^{\alpha} u={ }_{0} D_{t}^{\alpha} u,{ }_{t}^{c} D_{T}^{\alpha} u={ }_{t} D_{T}^{\alpha} u, t \in[0, T]$. This means that the left and right Riemann-Liouville fractional derivatives of order $\alpha$ are equivalent to the left and right Caputo fractional derivatives of order $\alpha$.

The properties of the fractional derivative spaces $E^{\alpha, p}$ and $E_{0}^{\alpha, p}$ are listed as the following lemma:

Lemma 2.1. Let $0<\alpha \leq 1$ and $1<p<\infty$.

1) Both the fractional spaces $E^{\alpha, p}$ and $E_{0}^{\alpha, p}$ are reflexive and separable Banach spaces.

2) For any $u \in E_{0}^{\alpha, p}$ we have

$$
{ }_{0}^{c} D_{t}^{\alpha} u(t)={ }_{0} D_{t}^{\alpha} u(t), \text { for any } t \in[0, T]
$$

3) $E_{0}^{\alpha, p} \rightarrow L^{p}[0, T]$ is continuous and

$$
\|u\|_{L^{p}} \leq \frac{T^{\alpha}}{\Gamma(\alpha+1)}\left\|{ }_{0} D_{t}^{\alpha} u\right\|_{L^{p}}
$$

4) Assume that $\alpha>\frac{1}{p}$ and the sequence $\left\{u_{n}\right\}$ converges weakly to $u$ in $E_{0}^{\alpha, p}$, i.e. $u_{n} \rightarrow u$. Then $\left\{u_{n}\right\}$ converges strongly to $u$ in $C[0, T]$, i.e.

$$
\left\|u_{n}-u\right\|_{\infty} \rightarrow 0, \text { as } n \rightarrow \infty
$$

Moreover, if $\frac{1}{p}+\frac{1}{q}=1$, then

$$
\|u\|_{\infty} \leq \frac{T^{\alpha-1 / p}}{\Gamma(\alpha)((\alpha-1) q+1)^{1 / q}}\left\|_{0} D_{t}^{\alpha} u\right\|_{L^{p}}
$$

By the property (3) in Lemma 2.1, we observe that the equivalent norm in $E_{0}^{\alpha, p}$ is defined by $\|u\|_{\alpha, p}=\left(\int_{0}^{T}\left|{ }_{0}^{c} D_{t}^{\alpha} u(t)\right|^{p} d t\right)^{1 / p}, \forall u \in E_{0}^{\alpha, p}$. 
In this paper, the work space for problem (1.3) is $E^{\alpha}=E^{\alpha, 2}$ with $\frac{1}{2}<\alpha \leq 1$. The space $E^{\alpha}$ is a Hilbert space with the inner product and the corresponding norm defined by

$$
\langle u, v\rangle=\int_{0}^{T} D_{t}^{c} u(t) \cdot{ }_{0}^{c} D_{t}^{\alpha} v(t) d t \text { and }\|u\|_{\alpha}=\|u\|_{\alpha, 2}=\left(\left.\left.\int_{0}^{T}\right|_{0} ^{c} D_{t}^{\alpha} u(t)\right|^{2} d t\right)^{1 / 2}
$$

\section{Fractional forced pendulum}

In this section we deal with the fractional boundary value problem

$$
\begin{gathered}
-{ }_{t} D_{T 0}^{\alpha} D_{t}^{\alpha} u(t)+g(u(t))=f(t), \quad t \in(0, T) \\
u(0)=u(T)=0
\end{gathered}
$$

Where $\frac{1}{2}<\alpha<1, g \in C(\mathbb{R}, \mathbb{R})$, bounded and $f \in C[0, T]$.

We recall the notion of solution for (3.1).

Definition 3.1. A function $u:[0, T] \rightarrow \mathbb{R}$ is called a solution of (3.1) if

1. ${ }_{t} I_{T}^{1-\alpha} u\left({ }_{0} D_{t}^{\alpha} u(t)\right)$ and ${ }_{0} I_{t}^{1-\alpha} u(t)$ are derivable in $t \in(0, T)$ and

2. $u$ satisfies (3.1).

Moreover, associated to (3.1) we have the functional $I: E^{\alpha} \rightarrow \mathbb{R}$ defined by

$$
I(u)=\int_{0}^{T}\left[\frac{\left|{ }_{0} D_{t}^{\alpha} u(t)\right|^{2}}{2}-G(u(t))+f(t) u(t)\right] d t
$$

where

$$
G(t)=\int_{0}^{t} g(s) d s
$$

and remember that $u$ is weak solution of (3.1) if $u$ is a critical point of the functional $I$. We recall our main theorem.

Theorem 3.1. Let $\frac{1}{2}<\alpha<1, g \in C(\mathbb{R}, \mathbb{R})$, bounded and $f \in C[0, T]$. The problem (1.3) has at least one solution.

The proof of Theorem 3.1 is divided into two parts. In the first part we prove the existence of $u \in E^{\alpha}$ such that

$$
I(u)=\min _{E^{\alpha}} I(v)
$$


for this purpose we use the Theorem 1.2. On the second part using Theorem 1.3, we just proved that

$$
I^{\prime}(u)=0 .
$$

First, we consider the following fractional boundary value problem:

$$
\begin{gathered}
-{ }_{t} D_{T{ }_{0}}^{\alpha} D_{t}^{\alpha} u(t)=f(t, u(t)) \\
u(0)=u(T)=0
\end{gathered}
$$

Where $f:[0, T] \times \mathbb{R} \rightarrow \mathbb{R}$ is continuous and when there exists $K>0$ such that its indefinite integral

$$
F(x, y)=\int_{0}^{y} f(x, s) d s
$$

satisfies the condition

$$
F(x, y) \leq \mathcal{K} \text { for all }(x, y) \in[0, T] \times \mathbb{R}
$$

Weak solutions of (3.2) are the critical points of the functional

$$
\begin{aligned}
& \varphi: E^{\alpha} \rightarrow \mathbb{R} \\
& u \rightarrow \varphi(u)=\int_{0}^{T}\left[\frac{\left|{ }_{0} D_{t}^{\alpha} u(t)\right|^{2}}{2}-F(t, u(t))\right] d t
\end{aligned}
$$

We have the following theorem.

Theorem 3.2. Let $\frac{1}{2}<\alpha \leq 1, f \in C([0, T] \times \mathbb{R}, \mathbb{R})$ such that its indefinite integral $F$ satisfies condition (3.3). Then the problem (3.2) has at least one weak solution.

Proof. By condition (3.3)

$$
\varphi(u)=\int_{0}^{T}\left[\frac{\left|{ }_{0} D_{t}^{\alpha} u(t)\right|^{2}}{2}-F(t, u(t))\right] d t \geq \frac{\|u\|_{\alpha}^{2}}{2}-T \mathcal{K}
$$

and the functional $\varphi$ is coercive. We now show that it is weakly lower semicontinuous. Let $\left(u_{n}\right) \in E^{\alpha}$ such that $u_{n} \rightarrow u$. Then, by the compact embedding of $E^{\alpha}$ into $C[0, T], u_{n} \rightarrow u$ uniformly on $[0, T]$. Furthermore, since

$$
\int_{0}^{T}\left[{ }_{0} D_{t}^{\alpha} u_{n}(t)-{ }_{0} D_{t}^{\alpha} u(t)\right]^{2} d t \geq 0
$$


we deduce

$$
\int_{0}^{T}\left({ }_{0} D_{t}^{\alpha} u_{n}(t)\right)^{2} d t \geq \int_{0}^{T} 2_{0} D_{t}^{\alpha} u_{n}(t)_{0} D_{t}^{\alpha} u(t) d t-\int_{0}^{T}\left({ }_{0} D_{t}^{\alpha} u(t)\right)^{2} d t
$$

and hence

$$
\begin{gathered}
\liminf _{n \rightarrow \infty} \int_{0}^{T}\left({ }_{0} D_{t}^{\alpha} u_{n}(t)\right)^{2} d t \geq \liminf _{n \rightarrow \infty}\left(\int_{0}^{T} 2{ }_{0} D_{t}^{\alpha} u_{n}(t){ }_{0} D_{t}^{\alpha} u(t) d t-\int_{0}^{T}\left({ }_{0} D_{t}^{\alpha} u(t)\right)^{2} d t\right) \\
\geq \int_{0}^{T}\left({ }_{0} D_{t}^{\alpha} u(t)\right)^{2} d t
\end{gathered}
$$

This implies

$$
\lim _{n \rightarrow \infty} \inf \varphi\left(u_{n}\right) \geq \varphi(u)
$$

Then $\varphi$ is weakly lower-semicontinuous on $E^{\alpha}$ and the existence of a minimum for $\varphi$ follows from Theorem 1.2.

We apply this theorem to (3.1). First, Lax-Milgram theorem shows that the linear problem

$$
\begin{aligned}
& { }_{{ }_{t}} D_{T}^{\alpha}{ }_{0} D_{t}^{\alpha} u(t)=f(t) \\
& u(0)=u(T)=0
\end{aligned}
$$

has a unique solution $U(x)$. Letting $u=U+v$, the problem (3.1) is reduced to the equivalent one

$$
\begin{aligned}
& { }_{-} D_{T}{ }_{T 0} D_{t}^{\alpha} v(t)+g(U(t)+v(t))=0 \\
& v(0)=v(T)=0
\end{aligned}
$$

Moreover, let $M=\max _{u \in \mathbb{R}} G(u)$, then

$$
G(u) \leq M, \forall u \in \mathbb{R}
$$

and the corresponding function $F$ given by

$$
F(t, u(t))=G(U(t)+v(t)) \leq \mathcal{M}
$$

satisfies assumption (3.3). Thus Problem (3.1) always has at least one solution by Theorem 3.2. That is, there exists $u \in E^{\alpha}$ such that

$$
\varphi(u)=\inf _{E^{\alpha}} \varphi(v)
$$


where

$$
\varphi(u)=\int_{0}^{T}\left[\frac{\left|{ }_{0} D_{t}^{\alpha} u(t)\right|^{2}}{2}-G(U(t)+u(t))\right] d t
$$

Finally we prove that $u$ is a critical point of $\varphi$. For this fact, first we prove some lemmas.

Lemma 3.1. $I \in C^{1}\left(E^{\alpha}, \mathbb{R}\right)$ and

$$
I^{\prime}(u) v=\int_{0}^{T}\left[{ }_{0} D_{t}^{\alpha} u(t)_{0} D_{t}^{\alpha} v(t)-g(u(t)) v(t)+f(t) v(t)\right] d t
$$

Proof. We only show that the functional $\phi: E^{\alpha} \rightarrow \mathbb{R}$ defined by

$$
\Phi(u)=\int_{0}^{T} G(u) d t
$$

is $C^{1}\left(E^{\alpha}, \mathbb{R}\right)$. We are going to prove that

$$
\lim _{\|v\|_{\alpha} \rightarrow 0} \frac{|r(v)|}{\|v\|_{\alpha}}=0
$$

where

$$
r(v)=\Phi(u+v)-\Phi(u)-\int_{0}^{T} g(u) v d t
$$

By the FTC we have

$$
G(u+v)-G(u)=\int_{0}^{1} \frac{d}{d x} G(u+x v) d x
$$

then

$$
\begin{aligned}
r(v) & =\int_{0}^{T}[G(u+v)-G(u)] d t-\int_{0}^{T} g(u) v d t \\
& =\int_{0}^{T}\left[\int_{0}^{1}(g(u+x v)-g(u)) v d x\right] d t
\end{aligned}
$$

Therefore

$$
|r(v)| \leq \int_{0}^{T}\left[\int_{0}^{1} g(u+x v)-g(u)|| v \mid d x\right] d t
$$


Now, since $|g(t)| \leq K, \forall t \in \mathbb{R}$ we have

$$
|g(u+x v)-g(u)|^{2} \leq 4 K^{2} \in L^{1}[0, T]
$$

So by Fubini theorem, Hölder inequality and Lemma 2.1 we get

$$
\begin{aligned}
|r(v)| & \leq \int_{0}^{1}\left[\iint_{0}^{T} g(u+x v)-g(u) \| v \mid d t\right] d x \\
& \leq \int_{0}^{1}\|g(u+x v)-g(u)\|_{L^{2}}\|v\|_{L^{2}} d x \\
& \leq \frac{T^{\alpha}}{\Gamma(\alpha+1)} \int_{0}^{1}\|g(u+x v)-g(u)\|_{L^{2}}\|v\|_{\alpha} d x
\end{aligned}
$$

then

$$
\frac{|r(v)|}{\|v\|_{\alpha}} \leq \frac{T^{\alpha}}{\Gamma(\alpha+1)} \int_{0}^{1}\|g(u+x v)-g(u)\|_{L^{2}} d x
$$

Now for each $n \in \mathbb{N}$, let

$$
\begin{aligned}
\theta_{n}:[0,1] & \rightarrow \mathbb{R} \\
x & \rightarrow \theta_{n}(x)=\left\|g\left(u+x v_{n}\right)-g(u)\right\|_{L^{2}}
\end{aligned}
$$

Therefore

$$
\frac{\left|r\left(v_{n}\right)\right|}{\left\|v_{n}\right\|_{\alpha}} \leq \frac{T^{\alpha}}{\Gamma(\alpha+1)} \int_{0}^{1} \theta_{n}(x) d x
$$

For each fixed $x$, we get

$$
\lim _{n \rightarrow \infty} \int_{0}^{T}\left|g\left(u+x v_{n}\right)-g(u)\right|^{2} d t=0
$$

this implies, for each fixed $x, \theta_{n}(x) \rightarrow 0, \forall x \in[0,1]$. On the other hand

$$
\begin{aligned}
0 & \leq \theta_{n}(x) \leq\left\|g\left(u+x v_{n}\right)\right\|_{L^{2}}+\|g(u)\|_{L^{2}} \\
& \leq 2 K T^{1 / 2} \in L^{1}[0, T]
\end{aligned}
$$

By the Lebesgue's dominated convergence theorem

$$
\lim _{n \rightarrow \infty} \int_{0}^{T} \theta_{n}(x) d x=0 \Rightarrow \theta_{n} \rightarrow 0 \text { in } L^{1}[0, T]
$$


Now we show that $\phi^{\prime}$ is continuous. Let $\left\{v_{n}\right\} \in E^{\alpha}$ such that $v_{n} \rightarrow 0$. By Lemma 2.1

$$
v_{n} \rightarrow 0 \text { in } L^{2}[0, T] \text { and } v_{n}(x) \rightarrow 0 \text { a.e. on }[0, T]
$$

By the continuity of $g$

$$
g\left(\left(u+v_{n}\right)(t)\right) \rightarrow g(u(t)) \text { a.e. in }[0, T]
$$

this implies

$$
\left|g\left(\left(u+v_{n}\right)(t)\right)-g(u(t))\right|^{2} \rightarrow 0 \text { a.e. in }[0, T]
$$

On the other hand

$$
\left|g\left(\left(u+v_{n}\right)(t)\right)-g(u(t))\right|^{2} \leq(2 K)^{2} \in L^{1}[0, T]
$$

By Lebesgue's dominated convergence theorem

$$
\lim _{n \rightarrow \infty} \int_{0}^{T}\left|g\left(\left(u+v_{n}\right)(t)\right)-g(u(t))\right|^{2} d t=0
$$

Now by Hölder inequality and Lemma 2.1, we get

$$
\begin{aligned}
\left|\left\langle\Phi^{\prime}\left(u+v_{n}\right)-\Phi^{\prime}(u), v\right\rangle\right| & =\left|\int_{0}^{T}\left(g\left(u+v_{n}\right)-g(u)\right) v d t\right| \\
& \leq\left(\int_{0}^{T}\left|g\left(u+v_{n}\right)-g(u)\right|^{2} d t\right)^{1 / 2}\left(\left.\int_{0}^{T} v\right|^{2} d t\right)^{1 / 2} \\
& \leq \frac{T^{\alpha}}{\Gamma(\alpha+1)}\left\|g\left(u+v_{n}\right)-g(u)\right\|_{L^{2}}\|v\|_{\alpha}
\end{aligned}
$$

This implies

$$
\begin{aligned}
\left\|\Phi^{\prime}\left(u+v_{n}\right)-\Phi^{\prime}(u)\right\|_{\left(E^{\alpha}\right)^{*}} & =\sup _{\|v\|_{\alpha} \leq 1}\left|\left\langle\Phi^{\prime}\left(u+v_{n}\right)-\Phi^{\prime}(u), v\right\rangle\right| \\
& \leq \frac{T^{\alpha}}{\Gamma(\alpha+1)}\left\|g\left(u+v_{n}\right)-g(u)\right\|_{L^{2}} \rightarrow 0
\end{aligned}
$$

as $n \rightarrow \infty$. Therefore $\Phi^{\prime}$ is continuous. 
Lemma 3.2. $I$ satisfies the $(P S)_{c}$ - condition.

Proof. For this fact, we use the equivalent problem (3.6), associated to this problem, we have the functional

$$
\varphi(u)=\int_{0}^{T}\left[\frac{\left|{ }_{0} D_{t}^{\alpha} u(t)\right|^{2}}{2}-G(U+u)\right] d t
$$

$\varphi \in C^{1}\left(E^{\alpha}, \mathbb{R}\right)$ and is bounded below. If $u_{n} \in E^{\alpha}$ is a sequence such that

$$
\varphi\left(u_{n}\right) \rightarrow c \text { and } \varphi^{\prime}\left(u_{n}\right) \rightarrow 0 \text { as } n \rightarrow \infty
$$

We claim $u_{n}$ is bounded in $E^{\alpha}$. For $n$ large enough and (3.4) we get

$$
c+1 \geq \varphi\left(u_{n}\right) \geq \frac{1}{2}\left\|{ }_{0} D_{t}^{\alpha} u_{n}\right\|_{L^{2}}^{2}-T \mathcal{M}
$$

Therefore $u_{n}$ is bounded in $E^{\alpha}$ and hence, up to a subsequence, weakly converges to some $u \in E^{\alpha}$ and uniformly to $u$ on $[0, T]$. Consequently

$$
\lim _{n \rightarrow \infty}\left\langle\varphi^{\prime}\left(u_{n}\right)-\varphi^{\prime}(u), u_{n}-u\right\rangle=0
$$

But

$$
\begin{aligned}
& \left\langle\varphi^{\prime}\left(u_{n}\right)-\varphi^{\prime}(u), u_{n}-u\right\rangle=\left\langle\varphi^{\prime}\left(u_{n}\right), u_{n}-u\right\rangle-\left\langle\varphi^{\prime}(u), u_{n}-u\right\rangle \\
& \int_{0}^{T} D_{t}^{\alpha} u_{n}(t)_{0} D_{t}^{\alpha}\left(u_{n}-u\right)(t)-G^{\prime}\left(U+u_{n}\right)\left(u_{n}-u\right)(t) d t \\
& -\int_{0}^{T} D_{t}^{\alpha} u(t)_{0} D_{t}^{\alpha}\left(u_{n}-u\right)(t)-G^{\prime}(U+u)\left(u_{n}-u\right)(t) d t \\
& =\int_{0}^{T}\left|D_{0} D_{t}^{\alpha} u_{n}(t)(t)\right|^{2} d t-\int_{0}^{T}\left(G^{\prime}\left(U+u_{n}\right)-G^{\prime}(U-u)\right)\left(u_{n}-u\right) d t
\end{aligned}
$$

Therefore

$$
\left\|u_{n}-u\right\|_{\alpha}^{2} \rightarrow 0 \text { as } n \rightarrow \infty
$$

and $(P S)_{c}$ condition is proved. 
Proof of Theorem 3.1. By the previous result we can apply Theorem 1.3 to get $\varphi^{\prime}(u)=0$. Hence we prove that there is $u \in E^{\alpha}$ weak solution of (3.1).

Now following the ideas of [30], since $u$ is a weak solution of (3.1) we can show that there exists a constant $C \in \mathbb{R}$ such that

$$
{ }_{t} I_{T}^{1-\alpha}{ }_{0} D_{t}^{\alpha} u(t)=-\int_{0}^{t} g(U(t)+v(t)) d t+C
$$

We notice that for $u \in E^{\alpha},{ }_{0} I_{t}^{1-\alpha} u(t)$ is derivable in $t \in(0, T)$ and

$$
\left({ }_{0} I_{t}^{1-\alpha} u(t)\right)^{\prime}={ }_{0} D_{t}^{\alpha} u(t) \in L^{2}[0, T]
$$

Moreover, by (3.10) we have

$$
\left({ }_{t} I_{T}^{1-\alpha}{ }_{0} D_{t}^{\alpha} u(t)\right)^{\prime} \in L^{1}[0, T]
$$

and

$$
{ }_{t} D_{T}^{\alpha}{ }_{0} D_{t}^{\alpha} u(t)=-\left({ }_{t} I_{T}^{1-\alpha}{ }_{0} D_{t}^{\alpha} u(t)\right)^{\prime}=g(U(t)+u(t))
$$

and since $u \in E^{\alpha}$ implies that $u(0)=u(T)=0$. The proof is complete.

\section{Acknowledgements}

C.T. was partially supported by MECESUP 0607.

\section{References}

[1] Kilbas A., Srivastava H., Trujillo J., Theory and Applications of Fractional Differential Equations, North-Holland Mathematics Studies, Amsterdam 2006.

[2] Miller K., Ross B., An Introduction to the Fractional Calculus and Fractional Differential Equations, Wiley and Sons, New York 1993.

[3] Podlubny I., Fractional Differential Equations, Academic Press, New York 1999.

[4] Agarwal R., Benchohra M., Hamani S., Boundary value problems for fractional differential equations, Georg. Math. J. 2009, 16, 3, 401-411.

[5] Agarwal R., Belmekki M., Benchohra M., A survey on semilinear differential equations and inclusions involving Riemann-Liouville fractional derivative, Adv. Difference Equ. 2009, 9.

[6] Agrawal O., Tenreiro Machado J., Sabatier J., Fractional Derivatives and Their Application: Nonlinear Dynamics, Springer-Verlag, Berlin 2004.

[7] Anh V., Mcvinish R., Fractional differential equations driven by Lévy noise, J. Appl. Math. and Stoch. Anal. 2003, 16, 2, 97-119.

[8] Benchohra M., Henderson J., Ntouyas S., Ouahab A., Existence results for fractional order functional differential equations with infinite delay, J. of Math. Anal. Appl. 2008, 338, 2, 1340-1350. 
[9] Hilfer R., Applications of Fractional Calculus in Physics, World Scientific, Singapore 2000.

[10] Lakshmikantham V., Vatsala A., Basic theory of fractional differential equations, Nonl. Anal. 2008, 69, 8, 2677-2682.

[11] Metsler R., Klafter J., The restaurant at the end of the random walk: recent developments in the description of anomalous transport by fractional dynamics, J. Phys. A 2004, 37, 161-208.

[12] Sabatier J., Agrawal O., Tenreiro Machado J., Advances in Fractional Calculus. Theoretical Developments and Applications in Physics and Engineering, Springer-Verlag, Berlin 2007.

[13] West B., Bologna M., Grigolini P., Physics of Fractal Operators, Springer-Verlag, Berlin 2003.

[14] Riewe F., Nonconservative Lagrangian and Hamiltonian mechanics, Phys. Rev. E 1996, 53, 1890-1899.

[15] Riewe F., Mechanics with fractional derivative, Phys. Rev. E 1997, 55, 3581-3592.

[16] Klimek M., Fractional sequential mechanics-models with symmetric fractional derivative, Czech. J. Phys. 2001, 51, 1348-1354.

[17] Klimek M., Lagrangean and Hamiltonian fractional sequential mechanics, Czech. J. Phys. 2002, 52, 1247-1253.

[18] Agrawal O., Formulation of Euler-Lagrange equations for fractional variational problems, J. Math. Anal. Appl. 2002, 272, 368-379.

[19] Agrawal O., Analytical schemes for a new class of fractional differential equations, J. Phys. A: Mathematical and Theoretical 2007, 40, 21, 5469-5477.

[20] Baleanu D., Trujillo J., On exact solutions of a class of fractional Euler-Lagrange equations, Nonlinear Dyn. 2008, 52, 331-335.

[21] Klimek M., Existence and uniqueness result for a certain equation of motion in fractional mechanics, Bull. Polish Acad. Sci. Tech. Sci. 2010, 58, 4, 573-581.

[22] Klimek M., Solutions of Euler-Lagrange equations in fractional mechanics, AIP Conference Proceedings 956. XXVI Workshop on Geometrical Methods in Physics, 2007, 73-78.

[23] Klimek M., G-Meijer functions series as solutions for certain fractional variational problem on a finite time interval, JESA 2008, 42, 653-664.

[24] Leszczynski S., Blaszczyk T., Modeling the transition between stable and unstable operation while emptying a silo, Granular Matter 2011, 13, 429-438.

[25] Szymanek E., The application of fractional order differential calculus for the description of temperature profiles in a granular layer, [in:] Theory \& Appl. of Non-integer Order Syst., W. Mitkowski et al. (eds.), LNEE 275, Springer Inter. Publ. Switzerland, 2013, 243-248.

[26] Mawhin J., Seventy-five years of global analysis around the forced pendulum equation, EQUADIFF 1997, 9, 115-145.

[27] Mawhin J., Global results for the forced pendulum equation, [in:] Handbook of Differential Equations, vol. 1, 2004, 533-589.

[28] Grossinho M., Tersian S., An Introduction to Minimax Theorems and Their Applications to Differential Equations, Kluwer Academic Publishers, London 2001.

[29] Bourdin L., Existence of a weak solution for fractional Euler-Lagrange equations, J. Math. Anal. Appl. 2013, 399, 239-251.

[30] Jiao F., Zhou Y., Existence results for fractional boundary value problem via critical point theory, Intern. Journal of Bif. and Chaos 2012, 22, 4, 1-17.

[31] Agrawal O., A general formulation and solution scheme for fractional optimal control problems, Nonlinear Dyn. 2004, 38, 323-337.

[32] Agrawal O., A numerical scheme and an error analysis for a class of Fractional Optimal Control problems, Proceedings of the ASME 2009, San Diego, California 2009.

[33] Agrawal O., A general finite element formulation for fractional variational problems, J. Math. Anal. Appl. 2008, 337, 1-12. 
[34] Atanackovic T., Stankovic B., On a class of differential equations with left and right fractional derivatives, ZAMM 2007, 87, 537-539.

[35] Baleanu D., Avkar T., Lagrangians with linear velocities within Riemann-Liouville fractional derivatives, Nuovo Cimento B 2004, 119, 73-79.

[36] Baleanu D., Golmankhaneh A., Nigmatullin R., Golmankhaneh A., Fractional Newtonian mechanics, Cent. Eur. J. Phys. 2010, 8, 1, 120-125.

[37] Baleanu D., Muslish S., Lagrangian formulation of classical fields within Riemann-Liouville fractional derivatives, Phys. Scr. 2005, 72, 119-121.

[38] Baleanu D., Fractional Hamiltonian analysis of irregular systems, Signal Process 2006, 86, 2632-2636.

[39] Blaszczyk T., Application of the Rayleigh-Ritz method for solving fractional oscillator equation, Scientific Research of the Institute of Mathematics and Computer Science 2009, 2, 29-36.

[40] Cresson J., Fractional embedding of differential operators and Lagrangian systems, J. Math. Phys. 2007, 48, 033504.

[41] Samko S., Kilbas A., Marichev O., Fractional Integrals and Derivatives: Theory and Applications, Gordon and Breach, New York 1993. 